\title{
Fluoroquinolone resistance mechanisms in an Escherichia coli isolate, HUE1, without quinolone resistance-determining region mutations
}

\section{Toyotaka Sato ${ }^{1}$, Shin-ichi Yokota ${ }^{2}$, Ikuo Uchida ${ }^{3}$, Torahiko Okubo ${ }^{1}$, Masaru Usui ${ }^{1}$, Masahiro Kusumoto ${ }^{4}$, Masato Akiba ${ }^{4}$, Nobuhiro Fujiii ${ }^{2}$ and Yutaka Tamura ${ }^{1 *}$}

\author{
Laboratory of Food Microbiology and Food Safety, Department of Health and Environmental Sciences, School of Veterinary Medicine, Rakuno Gakuen University, \\ Ebetsu, Japan \\ 2 Department of Microbiology, Sapporo Medical University School of Medicine, Sapporo, Japan \\ ${ }^{3}$ Dairy Hygiene Research Division, Hokkaido Research Station, National Institute of Animal Health, Sapporo, Japan \\ ${ }^{4}$ Bacterial and Parasitic Disease Research Division, Safety Research Team, National Institute of Animal Health, Ibaraki, Japan
}

\section{Edited by:}

Kunihiko Nishino, Osaka University, Japan

Reviewed by:

Axel Cloeckaert, Institut National de la Recherche Agronomique, France Junichi Yamagishi, Nihon

Pharmaceutical University, Japan

*Correspondence:

Yutaka Tamura, Laboratory of Food Microbiology and Food Safety,

Department of Health and

Environmental Sciences, School of

Veterinary Medicine, Rakuno

Gakuen University, 582

Midorimachi-Bunkyoudai,

Ebetsu 069-8501, Japan.

e-mail: tamuray@rakuno.ac.jp
Fluoroquinolone resistance can cause major clinical problems. Here, we investigated fluoroquinolone resistance mechanisms in a clinical Escherichia coli isolate, HUE1, which had no mutations quinolone resistance-determining regions (QRDRs) of DNA gyrase and topoisomerase IV. HUE1 demonstrated MICs that exceeded the breakpoints for ciprofloxacin, levofloxacin, and norfloxacin. HUE1 harbored oqxAB and qnrS1 on distinct plasmids. In addition, it exhibited lower intracellular ciprofloxacin concentrations and higher mRNA expression levels of efflux pumps and their global activators than did reference strains. The genes encoding AcrR (local AcrAB repressor) and MarR (MarA repressor) were disrupted by insertion of the transposon IS3-IS629 and a frameshift mutation, respectively. A series of mutants derived from HUE1 were obtained by plasmid curing and gene knockout using homologous recombination. Compared to the MICs of the parent strain HUE1, the fluoroquinolone MICs of these mutants indicated that qnrS1, oqxAB, acrAB, acr $F, a c r D, m d t K, m d f A$, and tol $C$ contributed to the reduced susceptibility to fluoroquinolone in HUE1. Therefore, fluoroquinolone resistance in HUE1 is caused by concomitant acquisition of $\mathrm{QnrS} 1$ and $\mathrm{OqxAB}$ and overexpression of $\mathrm{Acr} A \mathrm{~B}-\mathrm{TolC}$ and other chromosome-encoded efflux pumps. Thus, we have demonstrated that QRDR mutations are not absolutely necessary for acquiring fluoroquinolone resistance in E. coli.

Keywords: AcrAB, efflux pump, Escherichia coli, fluoroquinolone resistance, oqxAB, qnrS

\section{INTRODUCTION}

Fluoroquinolones are widely used in the clinical treatment of various bacterial infections, such as urinary tract and blood stream infections caused by Escherichia coli. Many studies have reported the isolation of fluoroquinolone-resistant strains (Peña et al., 1995; Cizman et al., 2001; Sanchez et al., 2012). Fluoroquinolone resistance is mainly caused by point mutations in the quinolone resistance-determining regions (QRDRs) of the DNA gyrase (encoded by gyrA and $g y r B$ ) and topoisomerase IV (encoded by parC and parE) subunits (Yoshida et al., 1991; Conrad et al., 1996; Heisig, 1996; Breines et al., 1997). A slight decrease in susceptibility to fluoroquinolones is attributed to a single mutation in $\mathrm{gyrA}$. Secondary mutations in $g y r A$ and additional mutations in parC and/or parE are required to exceed the breakpoint of the fluoroquinolone MIC (Conrad et al., 1996; Heisig, 1996; Breines et al., 1997).

Recently, we reported a fluoroquinolone-resistant E. coli isolate without QRDR mutations, named HUE1 (Sato et al., 2011). Its MICs for fluoroquinolones, such as ciprofloxacin (CIP) and levofloxacin (LVX), exceeded the breakpoints established by the Clinical and Laboratory Standards Institute (CLSI) (Clinical and Laboratory Standards Institute, 2011). HUE1 possesses two plasmid-mediated quinolone-resistant determinants (PMQRs), viz., oqxAB and qnrS. In this bacterium, OqxAB is a plasmidencoded efflux pump; however, the gene is present on the chromosomal DNA in most Klebsiella pneumoniae and Enterobacter cloacae strains (Bin Kim et al., 2009). The presence of this pump confers resistance to several antimicrobial agents, such as olaquindox (OLA), trimethoprim (TMP), and chloramphenicol (CHL), and decreases bacterial susceptibility to fluoroquinolones (Hansen et al., 2007). QnrS, on the other hand, is a member of the pentapeptide-repeat protein family that protects DNA gyrase (and probably also topoisomerase IV) from binding to fluoroquinolones, thereby decreasing fluoroquinolone susceptibility (Jacoby, 2005). However, acquisition of these PMQRs alone results in only a low level of fluoroquinolone resistance, with MICs that do not exceed the breakpoints for fluoroquinolones (Jacoby, 2005; Hansen et al., 2007). E. coli isolates lacking QRDR mutations in gyrA and parC and showing concomitant acquisition of oq $x A B$ and qnrS have previously been reported in China; however, these isolates did not exceed the breakpoint for CIP (Zhao et al., 2010).

Our previous findings suggested that the fluoroquinolone resistance of HUE1, which lacks QRDR mutations, is associated 
with not only with the presence of $o q x A B$ and qnrS but also with other fluoroquinolone-resistance mechanism(s) (Sato et al., 2011). In the current study, we investigated the fluoroquinoloneresistance mechanisms of the HUE1 strain.

\section{METHODS}

\section{BACTERIAL ISOLATES}

E. coli HUE1 had been isolated from the urinary catheter of a 77-year-old female patient at Hokkaido University Hospital (Sapporo, Japan) in 2007 (Sato et al., 2011). The somatic (O) serotype was determined by the slide agglutination test by using Escherichia coli $\mathrm{O}$ antisera (Denka Seiken, Tokyo, Japan), and the flagellar $(\mathrm{H})$ serotype was determined using reference sera obtained from the Statens Serum Institut (Hillerød, Denmark).

\section{SUSCEPTIBILITY TESTING AND GENETIC ANALYSIS}

Norfloxacin (NOR) was purchased from Sigma-Aldrich (St Louis, $\mathrm{MO}$ ). Other antibiotics were obtained as described previously (Sato et al., 2011). Susceptibility to fluoroquinolones [CIP, LVX, urifloxacin (URX), sitafloxacin (STX), and NOR], nalidixic acid (NAL), CHL, and TMP was determined by the agar plate dilution method, according to CLSI guidelines (Clinical and Laboratory Standards Institute, 2011). Phe-Arg- $\beta$-naphthylamide (PAßN; final concentration, $20 \mathrm{mg} / \mathrm{L}$ ), which is an inhibitor of the resistance-nodulation-division (RND)-type efflux pump, was purchased from Sigma-Aldrich.
The presence of $o q x A, o q x B$, and $q n r S$ was determined by PCR (Sorensen et al., 2003; Cattoir et al., 2007). Fulllength nucleotide sequences of oqxAB, qnrS, acrA, acr $B, \operatorname{acrR}$, acrE, acrF, acrS, tolC, soxS, soxR, and rob were determined by PCR and direct sequencing by using the primer pairs listed in Table 1. The nucleotide sequence of $\operatorname{mar} R$ was determined as previously described (Lindgren et al., 2003). Nucleotide sequences were determined using a BigDye Terminator v3.1 Cycle Sequencing Kit (Life Technologies, Carlsbad, CA), and sequencing was performed in a 3130 Genetic Analyzer (Life Technologies). The oq $x A B$ nucleotide sequence of HUE1 was submitted to GenBank (accession number AB601773). All gene sequences, except those of oqxAB and $q n r S$, were compared with those of Escherichia coli strain K12 substrain MG1655, which was deposited in GenBank (accession number U00096), as the reference strain.

\section{TRANSFORMATION OF PLASMIDS DERIVED FROM HUE1 INTO DH5 $\alpha$}

Plasmids were isolated from HUE1 as described previously (Kado and Liu, 1981). The plasmids were electroporated into E. coli DH5 $\alpha$ (Takara, Shiga, Japan) by using an ECM600 (BTX, San Diego, CA, USA) under the following conditions: voltage, $1.8 \mathrm{kV}$; capacitance, $25 \mu \mathrm{F}$; and resistance, $200 \mathrm{ohms}$. oq $x A B$ and/or qnrS transformants were screened using Muller-Hinton (MH) agar containing CIP, TMP, and CHL at concentrations ranging from 2- to 8 -fold of the respective MIC values.

Table 1 | Sequences of primers used for PCR and DNA sequencing in this study.

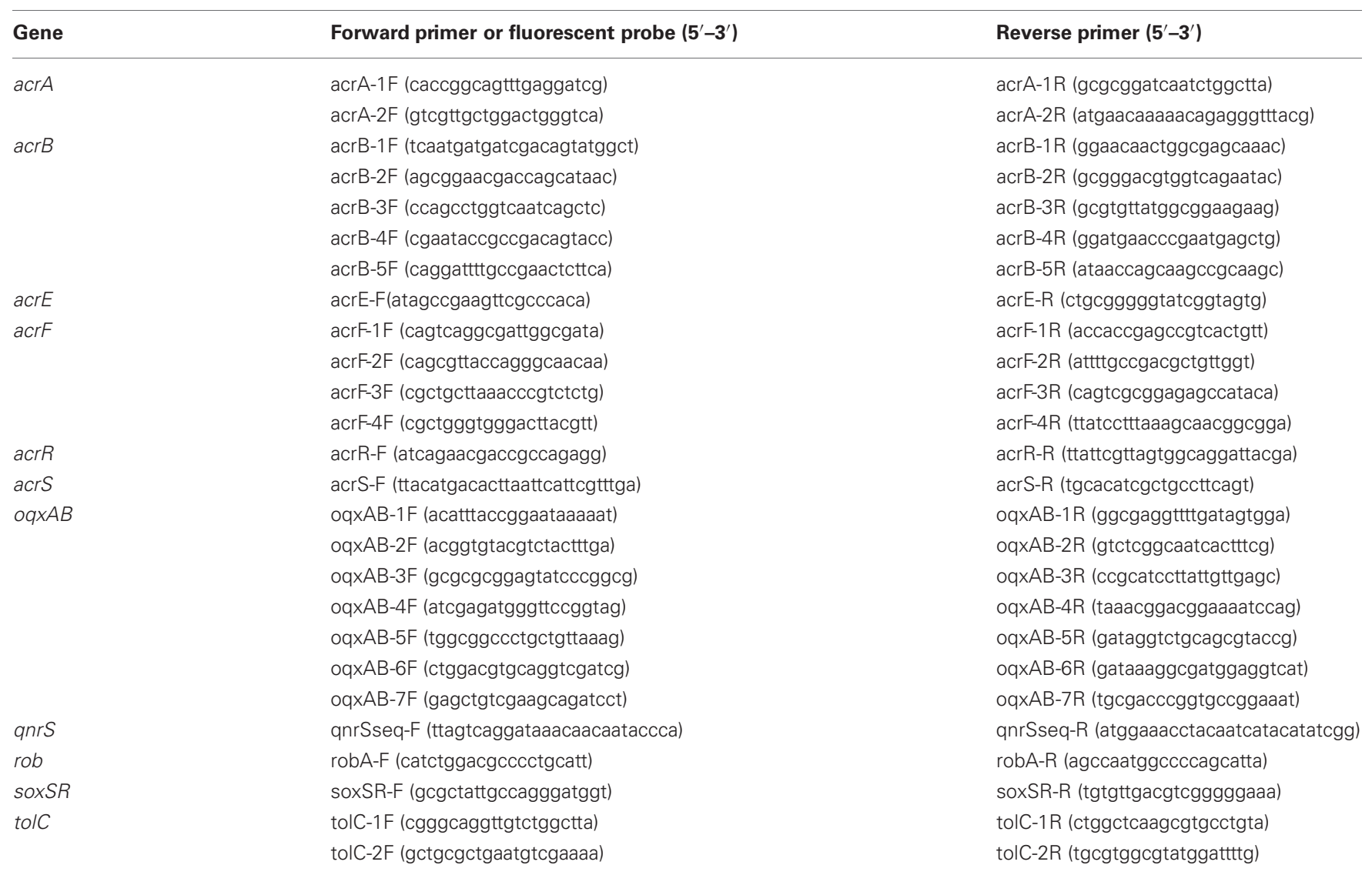




\section{PLASMID CURING, PLASMID RE-INTRODUCTION, AND SOUTHERN HYBRIDIZATION}

Curing of plasmids and generation of spontaneous mutants were performed as previously described, with slight modifications (Deane and Rawlings, 2004). Briefly, strains were grown in $4 \mathrm{~mL}$ $\mathrm{LB}$ broth at $40^{\circ} \mathrm{C}$ and incubated for periods ranging from a week to a month. Then, clones were picked and grown in $\mathrm{MH}$ agar containing sub-MIC concentrations of CIP, TMP, and CHL or no antimicrobials, in order to obtain oq $x A B$ - and/or qnrS-cured clones. Re-introduction of plasmids harboring $o q \times A B$ and/or $q n r S$ into plasmid-cured mutants was performed using electroporation as described above. The presence of $o q x A, o q x B$, and $q n r S$ was detected by PCR and Southern hybridization of plasmids, as previously described (Tamamura et al., 2011). Probes for oqxB and $q n r S 1$ were prepared by PCR by using specific primers, as described previously (Cattoir et al., 2007; Bin Kim et al., 2009). Probe labeling was carried out using a PCR DIG labeling mix (Roche Diagnostics, Tokyo, Japan).

\section{REAL-TIME REVERSE-TRANSCRIPTION (RT) PCR}

Overnight cultures were diluted 1:100 in LB broth and grown to the mid-logarithmic phase. RNA was isolated using an RNeasy Mini kit (Qiagen, Hilden, Germany). Gene expression was estimated by quantitative real-time reverse-transcription (RT)-PCR by using the probe and primer pairs shown in Table 2. RT-PCR was performed using a QuantiTect Probe RT-PCR kit (Qiagen) in $20 \mu \mathrm{L}$ reactions containing $2.5 \mathrm{ng}$ of purified RNA, $0.2 \mu \mathrm{M}$ of probe, and $0.5 \mu \mathrm{M}$ of each of the forward and reverse primers. The cycling conditions included reverse transcription at $50^{\circ} \mathrm{C}$ for $20 \mathrm{~min}$ and PCR involving initial activation at $95^{\circ} \mathrm{C}$ for $15 \mathrm{~min}$ and 45 cycles each consisting of $1 \mathrm{~min}$ at $55^{\circ} \mathrm{C}$ and $30 \mathrm{~s}$ at $60^{\circ} \mathrm{C}$, in a LightCycler 480 system (Roche, Mannheim, Germany). The E. coli strain AG100 (K-12 argE3 thi-1 rpsL xyl mtl D(gal-uvrB) supE44) (Okusu et al., 1996), which was gifted by Dr. Helen I. Zgurskaya (University of Oklahoma, USA), was used as a control. Expression levels of gapA were used to normalize expression ratios. Data, except for those of $o q x B$ and $q n r S 1$, were calibrated against expression levels in AG100, which were set as 1, to determine fold changes in expression. Data for $o q x B$ and $q n r S 1$ were calibrated to the respective levels in HUE1, which were set as 1 . The data shown represent the mean values of three independent experiments.

\section{CONSTRUCTION OF GENE DELETION MUTANTS}

Gene disruption was performed by Red/ET recombination by using the Quick and Easy Gene Deletion kit (Gene Bridges $\mathrm{GmbH}$, Heidelberg, Germany) according to the manufacturer's protocol. The relevant forward and reverse primers are shown in Table 2. Briefly, target gene-specific minigenes, containing a neomycin/kanamycin- or hygromycin-resistance cassette, were constructed by PCR with primers containing $46 \mathrm{bp}$ of upstream and downstream sequences that contained the start codon or the stop codon (or the last codon before the stop codon) of the target genes, respectively (Table 2 ). The target genes were replaced with minigenes containing the drug-resistance cassette by using Red/ET recombination. The integration of interrupted genes was verified by PCR and DNA sequencing.

\section{PLASMID CONSTRUCTION}

Each of the wild-type acrR and wild-type marR DNA segments were amplified by PCR using DH5 $\alpha$ genomic DNA as a template and primers containing the A1 T7 promoter, consensus Shine-Dalgarno, and HindIII restriction sites (Table 2), which were designed according to a previous report (Edgar et al., 2012). These PCR products were cloned into the HindIII site of pUC19 (wt-acrR or wt-marR), and the plasmids were then transformed into HUE1.

\section{ACCUMULATION ASSAYS}

Intracellular CIP concentrations were assayed using a fluorometric uptake assay (Usui et al., 2009). Forty milligrams of wet cells was treated with CIP (final concentration, $10 \mathrm{mg} / \mathrm{L}$ ) in the presence or absence of carbonyl cyanide $m$-chlorophenylhydrazone (CCCP, Sigma-Aldrich; final concentration, $150 \mu \mathrm{M}$ ). The fluorescence of CIP was measured at excitation and emission wavelengths of 277 and $445 \mathrm{~nm}$, respectively, by using an RF5000 fluorescence spectrophotometer (Shimadzu, Kyoto, Japan) (Nakaminami et al., 2010). The data shown represent the mean value \pm standard deviation values calculated from at least three independent experiments.

\section{STATISTICAL ANALYSIS}

Statistical significance was determined by the Student's $t$-test. Differences among more than three groups were determined using the Mann-Whitney $U$-test. A $P$-value of 0.05 or less was considered statistically significant.

\section{RESULTS CHARACTERISTICS OF HUE1 AND GENETIC ANALYSIS OF OqXAB AND OnrS}

HUE1 was identified as ST48 (according to the Max-PlanckInstitut für Infektionsbiologie database), phylogenetic group A (Sato et al., 2011), and O125:H37. Its NOR exceeded the breakpoints established by the CLSI, similarly with CIP and LVX previously tested (Sato et al., 2011). A 4421-bp DNA segment containing oq $x A B$ and a 647-bp DNA segment containing qnrS derived from HUE1 were sequenced. The sequence of $o q x A B$ was $100 \%$ identical to that of plasmid pOLA52 (accession number EU370913) in an E. coli isolate obtained from swine in Sweden (Hansen et al., 2004). The sequence of $q n r S$ was $100 \%$ identical to that of qnrS1 in Shigella flexneri (accession number AB187515) (Hata et al., 2005). Plasmid profiling and Southern blotting analysis showed that $o q x A B$ and $q n r S 1$ were located on 2 independent plasmids, pHFQ1 (>165 kb) and pHFQ2 (>100 kb), respectively (Figure 1, lane 1).

Introduction of pHFQ1 (carrying oq $x A B$ ) into $\mathrm{DH} 5 \alpha$ slightly increased the MICs for fluoroquinolones (except for LVX) and NAL, as compared with those for the host strain DH5 $\alpha$ (Table 3). Introduction of pHFQ2 (carrying qnrS1) resulted in higher MICs for fluoroquinolones and NAL than those seen for DH5 $\alpha /$ pHFQ1. However, introduction of both pHFQ1 and pHFQ2 into DH5 $\alpha$ did not increase the MICs beyond the breakpoints for fluoroquinolone. Deletion of oq $x A B$ or qnrS from the transformants by Red/ET recombination reverted MICs for fluoroquinolones to levels similar to those of DH5 $\alpha$. 


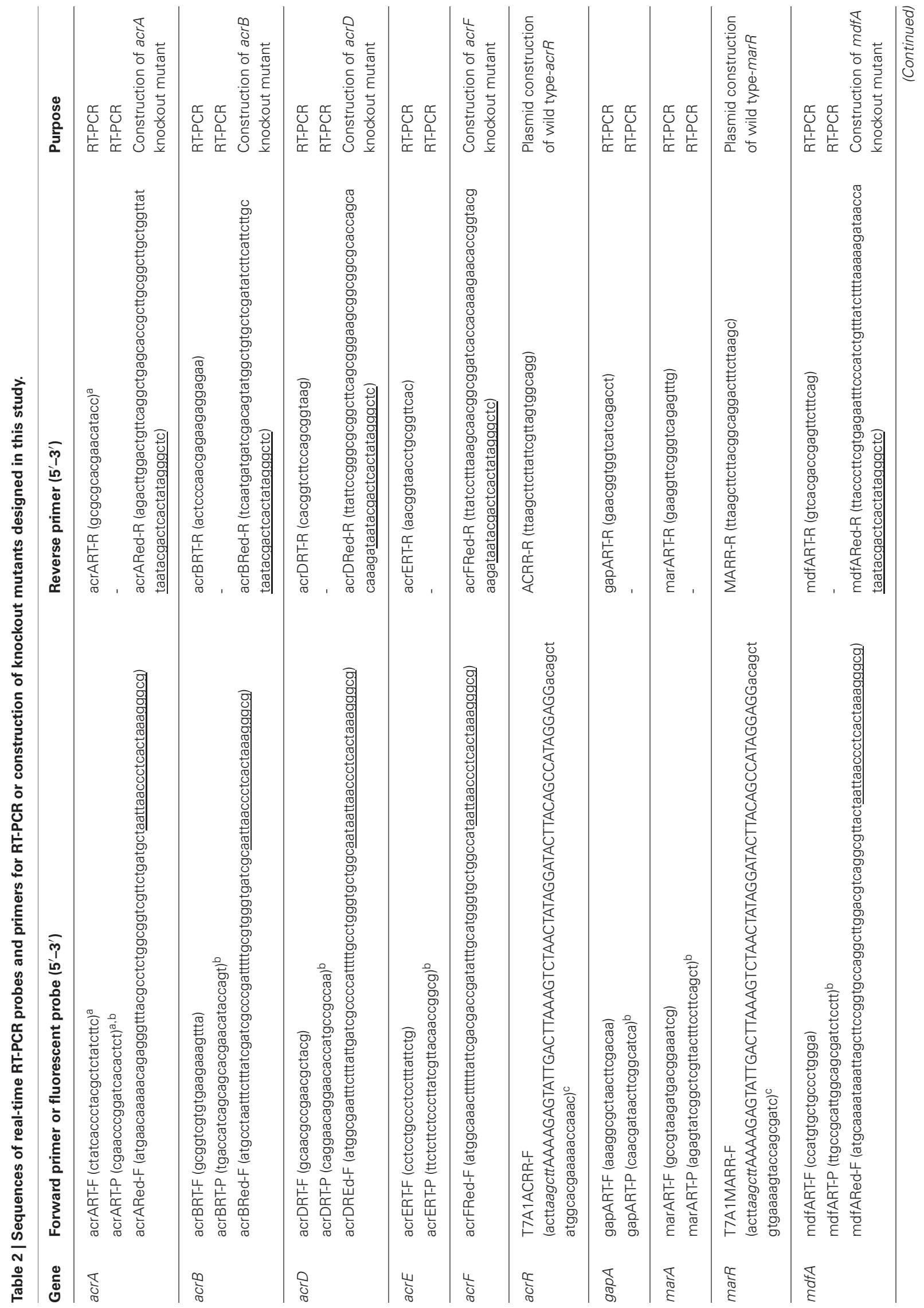




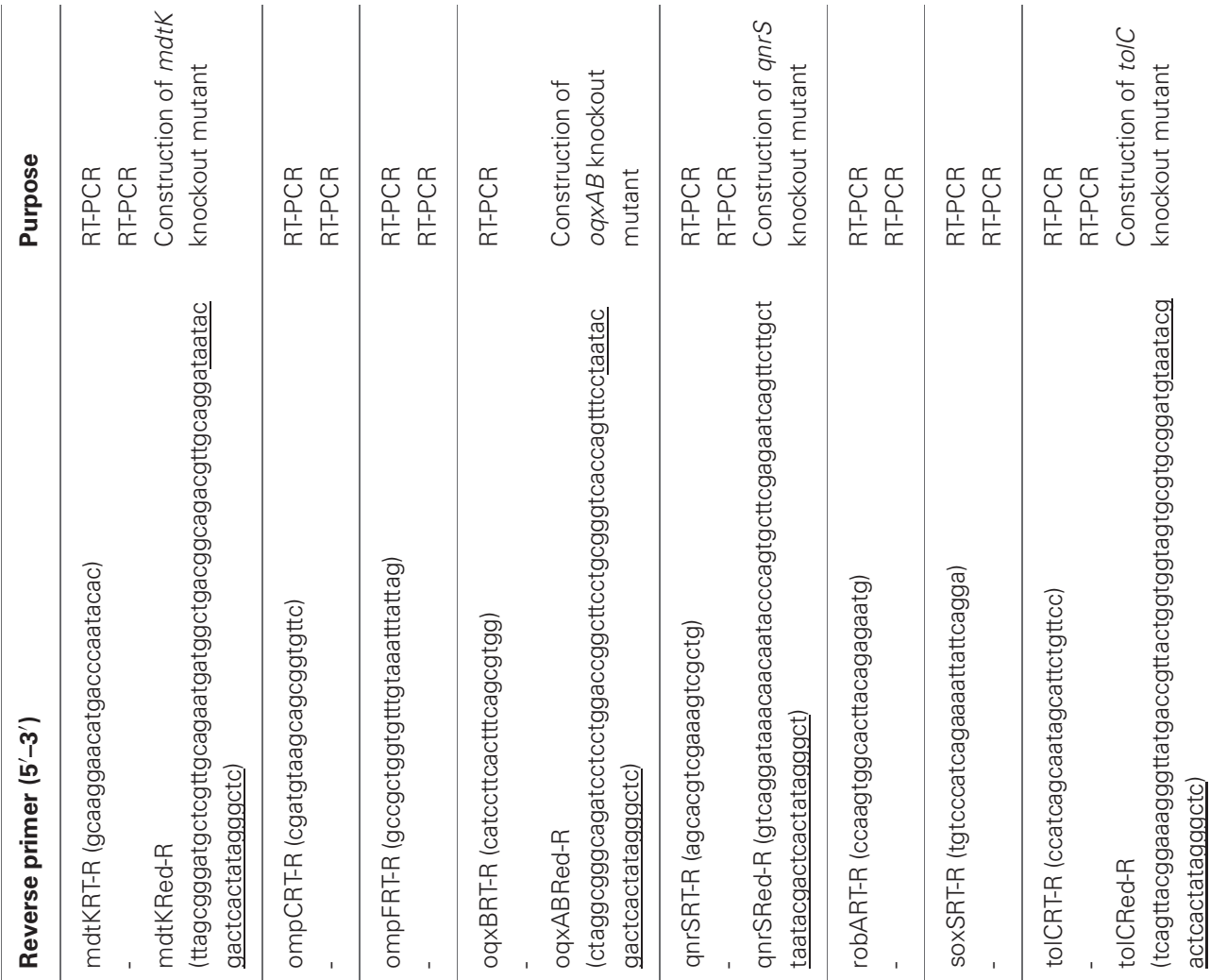

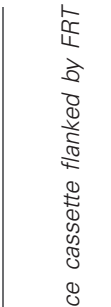
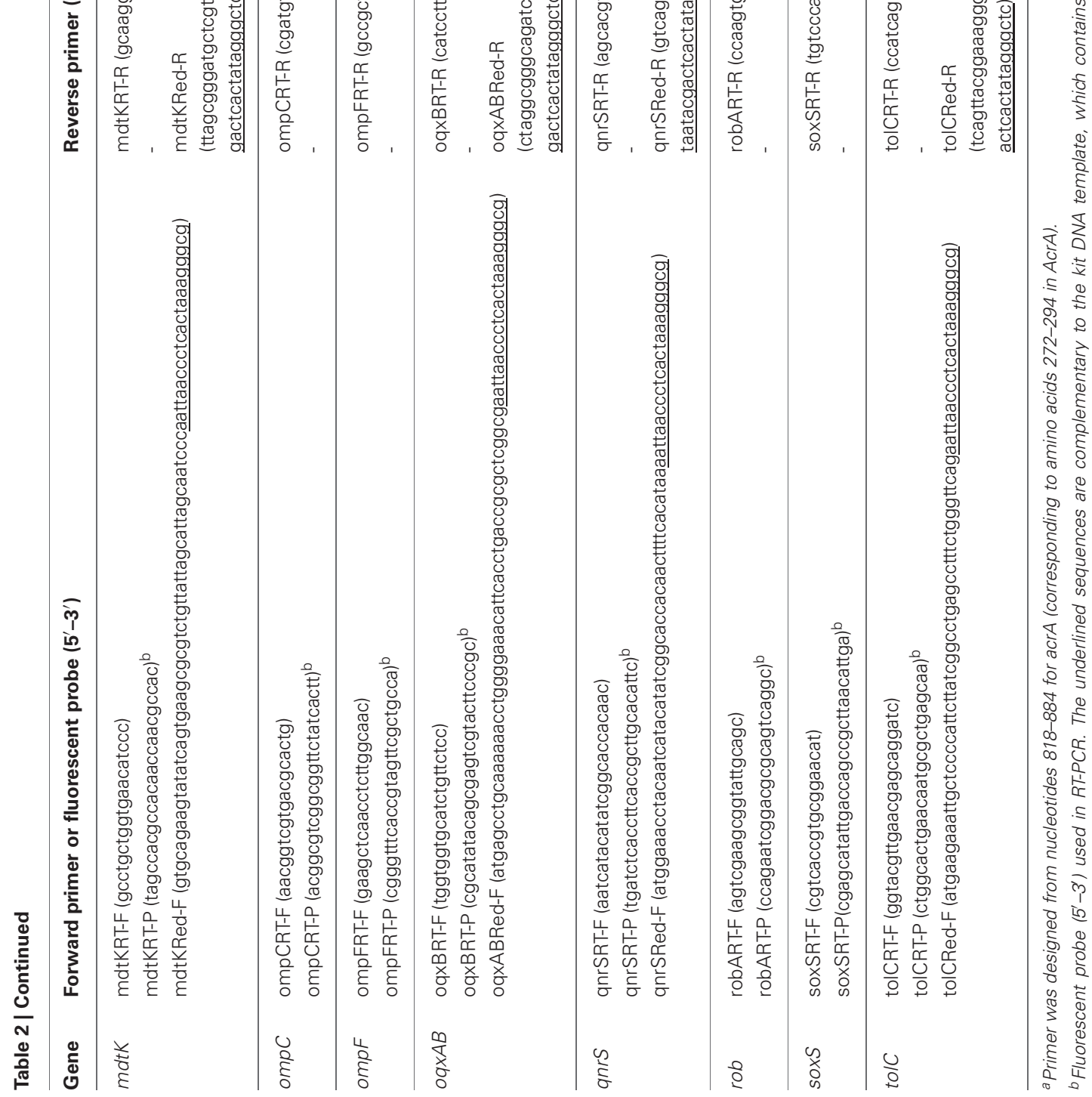

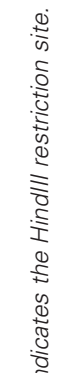

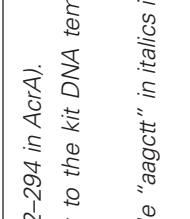

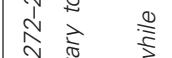

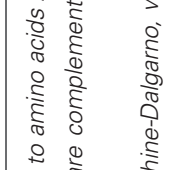

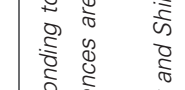

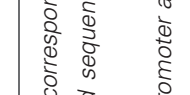

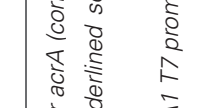

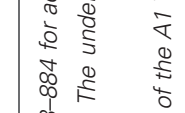

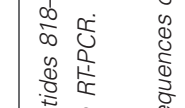

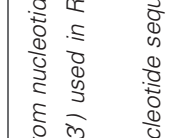

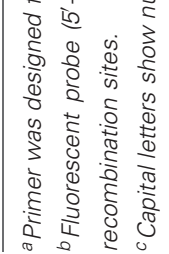




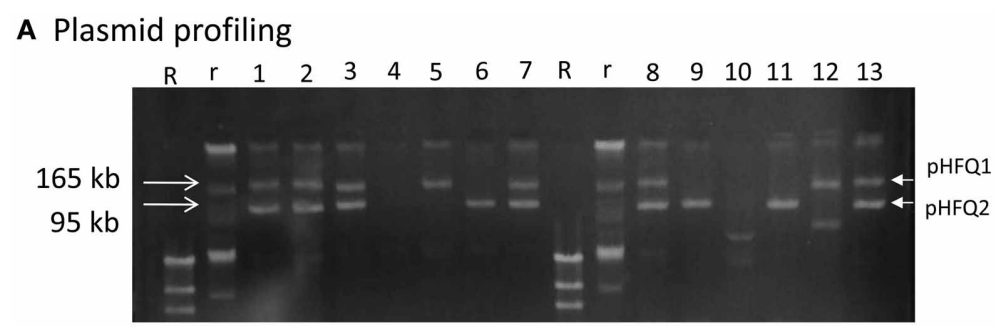

B oqxB

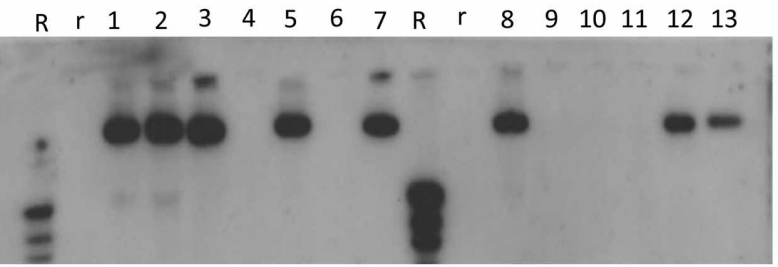

C qnrS1

$\begin{array}{lllllllllllllllll}\mathrm{R} & \mathrm{r} & 1 & 2 & 3 & 4 & 5 & 6 & 7 & \mathrm{R} & \mathrm{r} & 8 & 9 & 10 & 11 & 12 & 13\end{array}$

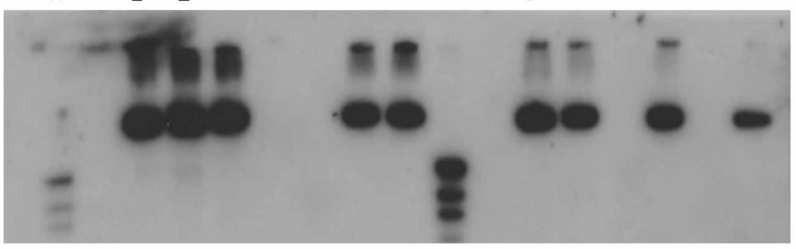

FIGURE 1 |Plasmid profile and Southern hybridization of oqxB and qnrS1 in HUE1, its oqxAB- and/or qnrS1-cured strains, and oqxAB- and/or qnrS1-re-introduced strains. (A) Plasmid profile. Electrophoresis was performed at $100 \mathrm{~V}$ for $70 \mathrm{~min}$ with a $0.8 \%$ agarose gel. (B) Southern hybridization with the oqxB probe. (C) Southern hybridization with the anrS probe. Lane 1, HUE1; lane
2. HUE1A2; lane 3, HUE1A; lane 4, HUE1A-Curoqxqnr; lane 5, HUE1A-Reoqx; lane 6, HUE1A-Reqnr; lane 7, HUE1A-Reoqxqnr; lane 8, HUE1; lane 9, HUE1B-Curoqx; lane 10, HUE1B-Curoqxqnr; lane 11, HUE1B-Reqnr; lane 12, HUE1B-Reoqx; lane 13, HUE1B-Reoqxqnr; R, DNA Molecular Weight MarkII, DIG-labeled: r, BAC-Tracker Supercoiled DNA Ladder.

\section{CHARACTERIZATION OF MUTANTS DERIVED FROM PLASMID CURING AND PLASMID RE-INTRODUCTION}

Eleven mutants obtained by curing and re-introduction of the plasmids showed altered fluoroquinolone MICs (Figure 1 and Table 3). These mutants were grouped into two types (groups $\mathrm{A}$ and $\mathrm{B}$ ). In the case of group A, we first obtained two strains, named HUE1A and HUE1A2. Although these two mutants still harbored pHFQ1 (carrying oqxAB) and pHFQ2 (carrying qnrS1; Figure 1, lanes 2 and 3), the MICs of NAL and fluoroquinolones were 2- or 4-fold lower than those of the parental strain, HUE1. Secondary screening of mutants by using HUE1A yielded a mutant, HUE1A-Curoqxqnr, which had lost pHFQ1 and pHFQ2 (Figure 1, lane 4). This mutant had 16- to 64fold lower MICs for fluoroquinolones than the parental strain, HUE1A. Re-introduction of pHFQ1 and pHFQ2 into HUE1ACuroqxqnr yielded HUE1A-Reoqxqnr, in which fluoroquinolone MICs recovered to the levels seen for HUE1A, but not to the MICs of the parent strain, HUE1.

In the case of group B, we first obtained a mutant, HUE1BCuroqx, which had lost pHFQ1; a second screening using HUE1B-Curoqx yielded HUE1B-Curoqxqnr, which had lost both pHFQ1 and pHFQ2 (Figure 1, lanes 9 and 10). Interestingly, the fluoroquinolone MICs of HUE1B-Curoqxqnr were 512- and 1280-fold lower than those of HUE1 and were 4- to 16-fold lower than those of HUE1A-Curoqxqnr (Table 3 ).

Similar differences with respect to the MICs of groups A and B were also observed for the mutant series in which pHFQ2 (carrying qnrS1) had been re-introduced. However, most fluoroquinolone MICs of group B strains in which pHFQ1 (carrying oq $x A B)$ had been re-introduced were only 2- to 4 -fold lower than those of mutants in group A.

\section{EFFECTS OF EFFLUX PUMP INHIBITORS AND GENETIC ANALYSIS OF EFFLUX PUMP COMPONENTS IN THE HUE1 STRAIN AND ITS MUTANTS}

The efflux pump inhibitor PA $\beta N$ reduced the fluoroquinolone MICs of the HUE1 strain from 4- to 16-fold (Table 3). In mutants derived from plasmid curing in groups $A$ and $B$, the effects of $\mathrm{PA} \beta \mathrm{N}$ were less than in the parental strain, HUE1. Remarkably, the fluoroquinolone MICs of three mutants that lost oq $x A B$ in group B (HUE1B-Curoqxqnr, HUE1B-Curoqx, and HUE1BReqnr) were barely affected by PA $\beta \mathrm{N}$.

HUE1 exhibited higher mRNA expression of efflux pump genes (acrA, acrB, acrE, acrD, $m d t K, m d f A$, and tolC) and their global activators (soxS, marA, and rob) than the control strains, AG100 and DH5 $\alpha$. In contrast, ompF expression was lower in HUE1 than in the control strains (Table 4). Moreover, 
Table 3 | Fluoroquinolones and NAL susceptibilities of HUE1, transformants derived from DH5 $\alpha$, and HUE1mutants derived from plasmid curing and reintroduction.

\begin{tabular}{|c|c|c|c|c|c|c|c|c|}
\hline Strain & \multicolumn{2}{|c|}{ PCR } & \multicolumn{6}{|c|}{ MIC (mg/L) } \\
\hline HUE1 & + & + & $4(\times 4)^{\mathrm{a}}$ & $8(\times 16)$ & $4(\times 4)$ & $2(\times 16)$ & 16 & 128 \\
\hline \multicolumn{9}{|c|}{ TRANSFORMATION OF pHFQ1 AND pHFQ2 } \\
\hline $\mathrm{DH} 5 \alpha$ & - & - & 0.015 & 0.03 & 0.015 & 0.004 & 0.03 & 32 \\
\hline $\mathrm{DH} 5 \alpha / \mathrm{pHFQ} 1$ & + & - & 0.03 & 0.03 & 0.03 & 0.015 & 0.06 & 64 \\
\hline DH5 $\alpha / p H F Q 2\left(\Delta q n r S 1::\right.$ hyg $\left.^{r}\right)$ & - & - & 0.015 & 0.03 & 0.015 & 0.004 & 0.03 & 32 \\
\hline DH5 $\alpha /$ pHFQ1-pHFO2 & + & + & 0.25 & 0.5 & 0.125 & 0.25 & 0.25 & $>128$ \\
\hline \multicolumn{9}{|c|}{ MUTANTS OBTAINED FROM PLASMID CURING } \\
\hline \multicolumn{9}{|l|}{$<$ Group A $>$} \\
\hline HUE1A and HUE1A2 & + & + & $2(\times 2)$ & $2(\times 4)$ & $2(\times 2)$ & $1(\times 8)$ & 8 & 64 \\
\hline HUE1A-Reoqxqnr & + & + & $2(\times 2)$ & $2(\times 4)$ & $2(\times 2)$ & $1(\times 8)$ & 8 & 64 \\
\hline \multicolumn{9}{|l|}{$<$ Group B > } \\
\hline HUE1B-Curoqx & - & + & $0.25(\times 1)$ & $0.125(\times 1)$ & $0.125(\times 1)$ & $0.03(\times 1)$ & 0.25 & 4 \\
\hline HUE1B-Curoqxqnr & - & - & $0.008(\times 1)$ & $0.008(\times 1)$ & $0.008(\times 1)$ & $0.004(\times 1)$ & 0.03 & 1 \\
\hline HUE1B-Reoqx & + & - & $0.125(\times 8)$ & $0.06(\times 8)$ & $0.03(\times 2)$ & $0.03(\times 8)$ & 0.25 & 16 \\
\hline HUE1B-Reqnr & - & + & $0.125(\times 1)$ & $0.125(\times 1)$ & $0.125(\times 1)$ & $0.03(\times 1)$ & 0.25 & 4 \\
\hline HUE1B-Reoqxqnr & + & + & $1(\times 4)$ & $1(\times 4)$ & $0.5(\times 2)$ & $0.25(\times 8)$ & 4 & 32 \\
\hline
\end{tabular}

${ }^{a}$ Reduction of MIC by PABN (-fold), 'resistance.

Table 4 | mRNA expression levels of global activators, efflux pumps, and ompF genes in HUE1, HUE1A, HUE1A-Curoqxqnr, and HUE1B-Curoqqnr.

\begin{tabular}{|c|c|c|c|c|c|c|c|c|c|c|c|}
\hline \multirow[t]{2}{*}{ Strain } & \multicolumn{11}{|c|}{ Expression level (relative amount of AG100; in terms of fold change) } \\
\hline & soxs & marA & rob & acrA & acrB & acrE & acrD & mdtK & mdfA & tolc & $\frac{\text { Porin }}{\text { ompF }}$ \\
\hline HUE1 & 9.80 & 147.77 & 8.17 & 4.67 & 6.81 & 8.70 & 8.34 & 6.43 & 6.76 & 2.83 & 0.19 \\
\hline HUE1A & 4.82 & 80.65 & 4.56 & 2.89 & 3.55 & 4.42 & 3.93 & 3.21 & 3.70 & 2.10 & 0.15 \\
\hline $\mathrm{DH} 5 \alpha$ & 1.83 & 21.84 & 1.44 & 1.28 & 1.29 & 0.69 & 0.59 & 0.76 & 1.17 & 1.62 & 2.78 \\
\hline
\end{tabular}

in HUE1A and HUE1A-Curoqxqnr, the mRNA expression of efflux pumps and their regulatory genes were approximately half of those in HUE1, while those of HUE1B-Curoqxqnr were similar to those in HUE1. The mRNA expression of qnrS1 and $\operatorname{oq} \times B$ was not significantly different among the HUE1, HUE1A, HUE1A-Reoqxqnr, and HUE1B-Reoqxqnr strains (data not shown).

Next, we determined the full DNA sequences of efflux pump genes ( $a c r A, a c r B, a c r E, a c r F$, and tolC) and their regulatory genes ( $\operatorname{marR}, a c r S$, soxS, soxR, rob, and acrR). Although most genes in
HUE1 and its plasmid-cured mutants were wild-type mutants, we found some mutations. acrR was disrupted by the insertion of a transposon, an IS3-IS629 element, in HUE1 and group A HUE1A-Curoqxqnr (Figure 2). HUE1B-Curoqxqnr (group B) had a deletion across $a c r R$ (corresponding to amino acids from Met-1 to Leu-73 of AcrR) and acrA (corresponding to amino acids from Met-1 to Asp-106 of AcrA), in addition to the insertion of the IS3-IS629 element. In addition, a nucleotide deletion of cytosine at position 223 of marR caused a frameshift in HUE1, HUE1A-Curoqxqnr, and HUE1B-Curoqxqnr. 
A E. coli K12 MG1655

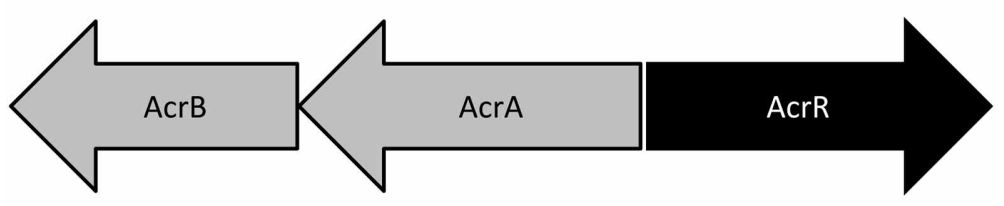

B HUE1 and HUE1A-Curoqxqnr

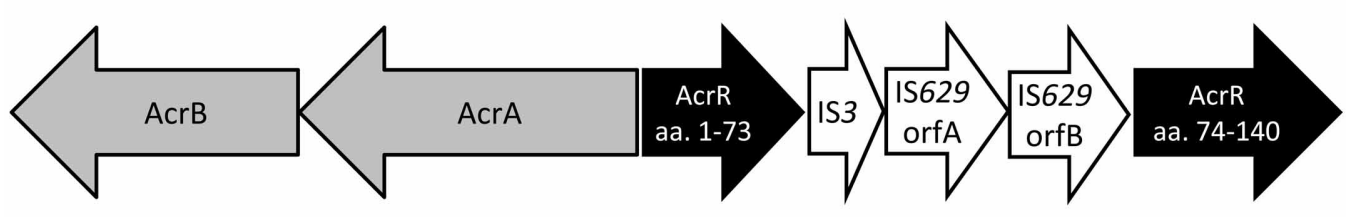

C HUE1B-Curoqxqnr

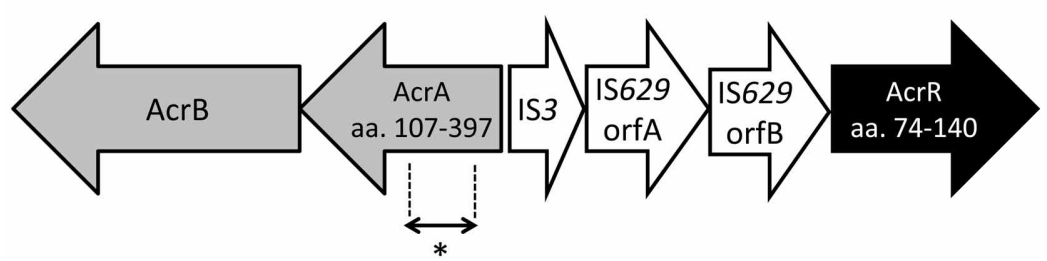

FIGURE 2 | Schematic representation of the acrAB-acrR region in HUE1, group A mutants, and group B mutants. (A) Escherichia coli K12 MG1655 (accession no U00096). (B) HUE1 and HUE1A-Curoqxanr (group A).
(C) HUE1B-Curoqxqnr (group B). aa., Amino acids. *Position of primer pairs (acrART-F and acrART-R) used in RT-PCR (corresponding to amino acids 272-294 in AcrA).

\section{CHARACTERIZATION OF KNOCKOUT MUTANTS PREPARED BY RED/ET RECOMBINATION}

We obtained a series of gene knockout mutants by Red/RT recombination. The fluoroquinolone MICs of HUE1- $\triangle$ oq $x A B$, HUE1- $\triangle q n r S 1$, and HUE1- $\triangle o q x A B$ qnrS1 were reduced by 2- or 4-fold, 16- or 32-fold, and 32- or 64-fold, as compared to those of HUE1, respectively (Table 5). HUE1- $\triangle$ acrA, HUE1- $\triangle a c r B$, and HUE1- $\triangle a c r A B$ demonstrated 4- or 8-fold lower MICs than HUE1. HUE1- $\triangle a c r A$ oq $x A B$ exhibited 32- or 64-fold lower fluoroquinolone MICs and HUE1- $\triangle a c r A$ qnrS1 exhibited 64- or 128fold lower fluoroquinolone MICs than HUE1. HUE1- $\triangle$ oq $x A B$ did not exhibit marked changes in fluoroquinolone MICs compared with those of HUE1, while HUE1- $\triangle a c r A B$ oq $x A B$ demonstrated larger fold changes, ranging from 4- to 16-fold lower, than HUE1- $\triangle a c r A B$.

Knockout of tolC markedly decreased fluoroquinolone MICs by 64 - or 128 -fold, and the MICs of the tolC knockout mutant (HUE1- $\triangle$ tolC) were not altered by additional knockout of $a c r A B$ or oq $x A B$ (HUE1- $\triangle$ tolC acrAB or HUE1- $\triangle$ tolC oq $x A B$ ). HUE1$\triangle$ tolC qnrS1 showed the lowest fluoroquinolone MICs, ranging from 512- to 2056-fold lower than those of HUE1. Mutants in which other efflux pump-associated genes, acrF, acrD, $m d t K$, and $m d f A$, were knocked out showed 2- to 4-fold lower fluoroquinolone MICs than did HUE1 (Table 5). The mutant derived from HUE1B-Curoqxqnr with knockout acrB (HUE1BCuroqxqnr- $\triangle a c r B$ ) did not show altered fluoroquinolone MICs, when compared to HUE1B-Curoqxqnr.

Transformation of HUE1 with plasmids encoding wild-type acrR or wild-type marR (HUE1-wt-acrR and HUE1-wt-marR) resulted in fluoroquinolone MICs that were 2- or 4-fold lower than those for HUE1 (data not shown). HUE1-wt-acrR and HUE1-wt-marR resulted in reduced mRNA expression levels of acr $A$ and $\operatorname{acr} B$, and HUE1-wt-marR also exhibited a significant reduction in the expression of marA compared with that for HUE1 (Figure 3).

\section{INTRACELLULAR FLUOROQUINOLONE CONCENTRATIONS}

The intracellular CIP concentration in HUE1 was approximately 2.3-fold lower than that in $\mathrm{DH} 5 \alpha(p<0.05$; Figure 4). Intracellular CIP concentrations in HUE1A and HUE1ACuroqxqnr were minimally, albeit significantly, higher than those of the parental strain, HUE1 $(p<0.05)$. In contrast, the intracellular CIP concentration in HUE1B-Curoqxqnr was markedly higher than those in HUE1, HUE1A, and HUE1A-Curoqxqnr $(p<0.05)$, and was similar to those of HUE1- $\triangle a c r A$ oq $x A B$, and HUE1- $\triangle a c r A B$ oqxAB (Figure 4). HUE1- $\triangle a c r A$ and HUE1$\triangle a c r A B$ exhibited significantly increased intracellular CIP concentrations compared with that for HUE1 $(p<0.05)$. However, the intracellular CIP concentrations of $a c r D-, a c r F-, ~ m d t K-$, and $m d f A$-knockout HUE1 mutants were slightly higher, but not significantly different from that for HUE1 $(p>0.05$; data not shown). The intracellular CIP concentration in HUE1- $\triangle$ oq $x A B$ was also not significantly different from that of HUE1; however, HUE1- $\triangle a c r A$ oq $x A B$ and HUE1- $\triangle a c r A B$ oq $x A B$ exhibited a clear increase in intracellular CIP levels compared to that in HUE1- $\triangle a c r A$ or HUE1- $\triangle a c r A B(p<0.05)$. HUE1- $\Delta$ tolC showed the highest intracellular CIP concentrations (Figure 4), which were not altered by additional knockout of $a c r A B$ or oq $x A B$ 
Table 5 | Fluoroquinolones susceptibilities of Red/ET recombination mutants derived from HUE1.

\begin{tabular}{|c|c|c|c|c|c|c|}
\hline \multirow[t]{2}{*}{ Strain } & \multicolumn{5}{|c|}{ MIC (mg/L) } & \multirow[t]{2}{*}{ MIC reduction (-fold) ${ }^{a}$} \\
\hline & CIP & LVX & URX & STX & NOR & \\
\hline HUE1 & 4 & 8 & 4 & 2 & 16 & - \\
\hline HUE1- $\triangle O q X A B$ & 2 & 2 & 1 & 0.5 & 8 & 2 or 4 \\
\hline HUE1- $\Delta q n r S 1$ & 0.25 & 0.25 & 0.125 & 0.125 & 0.5 & 16 or 32 \\
\hline HUE1- $\triangle$ oqxAB qnrS1 & 0.06 & 0.125 & 0.06 & 0.06 & 0.5 & 32 or 64 \\
\hline HUE1- $\triangle$ acrA & 1 & 1 & 0.5 & 0.25 & 4 & 4 or 8 \\
\hline HUE1- $\triangle$ acrA oqxAB & 0.125 & 0.125 & 0.125 & 0.03 & 0.25 & 32 or 64 \\
\hline HUE1- $\triangle$ acrA qnrS1 & 0.06 & 0.06 & 0.03 & 0.03 & 0.25 & 64 or 128 \\
\hline HUE1- $\triangle a c r B$ & 1 & 1 & 0.5 & 0.25 & 4 & 4 or 8 \\
\hline HUE1- $\triangle a c r A B$ & 1 & 1 & 0.5 & 0.25 & 4 & 4 or 8 \\
\hline HUE1- $\triangle a c r A B$ oq $x A B$ & 0.125 & 0.125 & 0.125 & 0.03 & 0.25 & 32 or 64 \\
\hline HUE1- $\triangle a c r A B$ qnrS1 & 0.06 & 0.06 & 0.03 & 0.03 & 0.5 & $32-128$ \\
\hline HUE1- $\Delta$ tolC & 0.06 & 0.125 & 0.06 & 0.015 & 0.25 & 64 or 128 \\
\hline HUE1- $\Delta$ tolC acrAB & 0.06 & 0.125 & 0.125 & 0.03 & 0.25 & 32 or 64 \\
\hline HUE1- $\triangle$ tolC oqxAB & 0.06 & 0.125 & 0.125 & 0.03 & 0.25 & 32 or 64 \\
\hline HUE1- $\Delta$ tolC qnrS1 & 0.002 & 0.004 & 0.008 & 0.002 & 0.015 & 512-2056 \\
\hline HUE1B-Curoqxqnr & 0.008 & 0.008 & 0.008 & 0.004 & 0.03 & 512 or 1024 \\
\hline HUE1B-Curoqxqnr- $\Delta a c r B$ & 0.008 & 0.008 & 0.008 & 0.004 & 0.03 & 512 or 1024 \\
\hline HUE1- $\triangle a c r F$ & 2 & 2 & 2 & 1 & 8 & 2 or 4 \\
\hline HUE1- $\triangle a c r D$ & 2 & 4 & 2 & 1 & 8 & 2 \\
\hline HUE1- $\Delta m d t K$ & 2 & 4 & 1 & 1 & 8 & 2 or 4 \\
\hline HUE1- $\Delta m d f A$ & 2 & 4 & 2 & 1 & 8 & 2 or 4 \\
\hline
\end{tabular}

${ }^{a}$ Relative value of HUE1.

(HUE1- $\triangle$ tolC acrAB or HUE1- $\triangle$ tolC oqxAB; data not shown). Addition of CCCP resulted in increased intracellular CIP concentrations in all strains, with levels ranging from $21.8 \pm 2.3$ to $25.8 \pm 5.2 \mathrm{ng} / \mathrm{mg}$ wet cells; there were no statistical differences $(p>0.05$, data not shown).

\section{DISCUSSION}

It is known that QRDR mutations are required for the fluoroquinolone MICs to exceed the breakpoints (Jacoby, 2005; Hansen et al., 2007; Cesaro et al., 2008; Zhao et al., 2010). To our knowledge, a fluoroquinolone-resistant strain without QRDR mutations has been reported only in a laboratory-derived resistant mutant strain of Acinetobacter baumannii that had been selected by in vitro fluoroquinolone exposure experiments; however, the resistance mechanism of this strain remains unclear (Chopra and Galande, 2011). In the current study, we demonstrated the fluoroquinolone-resistance mechanisms in an E. coli clinical isolate, HUE1, which lacks mutations in QRDRs (Sato et al., 2011).

HUE1 possessed two PMQRs on different plasmids, pHFQ1 (harboring oqxAB) and pHFQ2 (harboring qnrS1). Transformation experiments using $\mathrm{DH} 5 \alpha$ as a host clearly showed that oq $x A B$ and qnrS1 did in fact contribute to fluoroquinolone resistance. However, transformants containing both plasmids still did not exceed the MIC breakpoints.

It has been suggested that some efflux pumps are associated with fluoroquinolone resistance in HUE1. AcrAB-TolC is known to be the most important chromosomally encoded efflux pump related to fluoroquinolone resistance in Escherichia coli (Poole, 2005). During plasmid curing, a series of HUE1 group B mutants showed markedly lower fluoroquinolone MICs and higher intracellular CIP concentrations than did HUE1 and its group A mutants, whereas these mutants had mRNA expression levels of efflux pumps similar to those of HUE1. We found that group B mutants possessed deletions across parts of acrA. AcrA is an outer membrane protein that binds to AcrB (which plays a role in fluoroquinolone excretion) and TolC (an outer membrane factor) (Kobayashi et al., 2001; Elkins and Nikaido, 2003; Ge et al., 2009). The deleted region of AcrA in group B mutants involves an $\alpha-\beta$ barrel (53-61 amino acid residues), which forms the AcrB-binding domain, and a part of an $\alpha$-helical hairpin (99172 amino acid residues), which forms the TolC-binding domain (Ge et al., 2009). Fluoroquinolone MICs of the $\triangle a c r A$-knockout HUE1 mutant (HUE1- $\triangle a c r A$ ) were similar to those of HUE1BReoqxqnr (a group B mutant that possesses oqxAB and qnrS1), HUE1- $\triangle a c r B$, and HUE1- $\triangle a c r A B$. Moreover, the intracellular CIP concentration in HUE1B-Curoqxqnr (a group B mutant that had been cured of $o q x A B$ and qnrS1) was not different from that in HUE1B-Curoqxqnr- $\triangle a c r B$. These results suggested that reduction of fluoroquinolone MICs in group B strains was caused by functional disruption of AcrAB-TolC, which did not allow cooperation between AcrA and AcrB and TolC, indicating that fluoroquinolone resistance in HUE1 is partially due to AcrAB-TolC. 


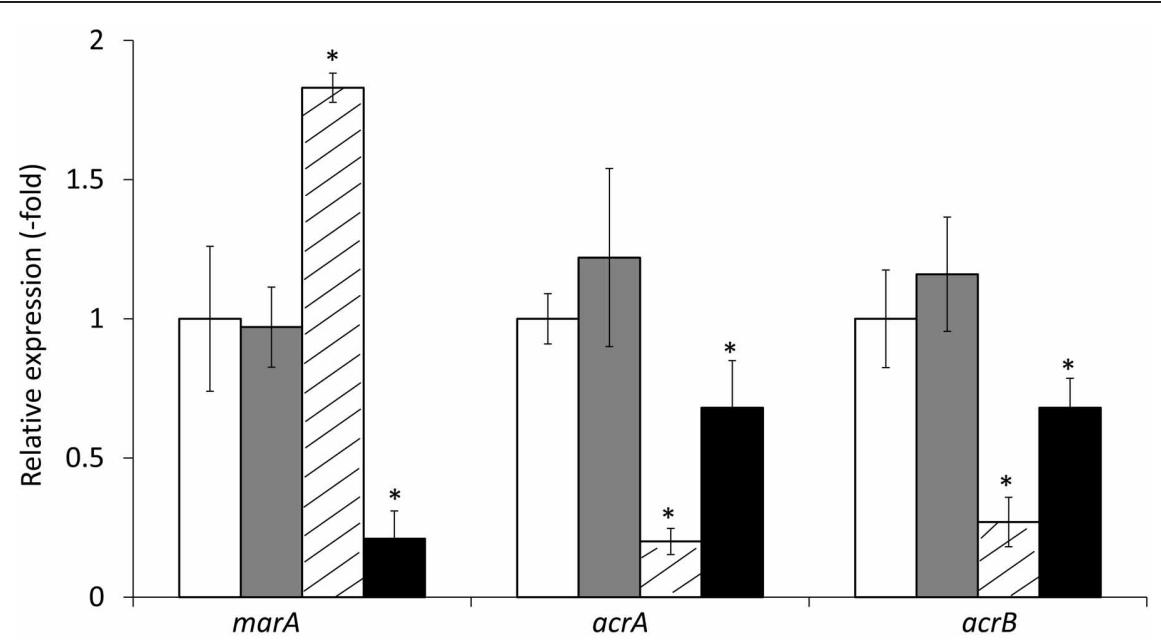

FIGURE 3 | mRNA expression levels of $\operatorname{mar} A$, acrA, and acrB in HUE1 and its acr $\boldsymbol{R}$ or $\boldsymbol{m a r} \boldsymbol{R}$ transformants. The values indicate the expression levels of indicated genes relative to those in HUE1 (fold-changes). White, HUE1; gray, HUE1-pUC19; diagonal line, HUE1-wt-acrR (HUE1 strain transformed wild-type of acrR); black, HUE1-wt-marR (HUE1 strain transformed wild-type marR). ${ }^{*} p<0.05$ compared to HUE1. Data represent the mean \pm standard deviation values calculated from at least three independent experiments.

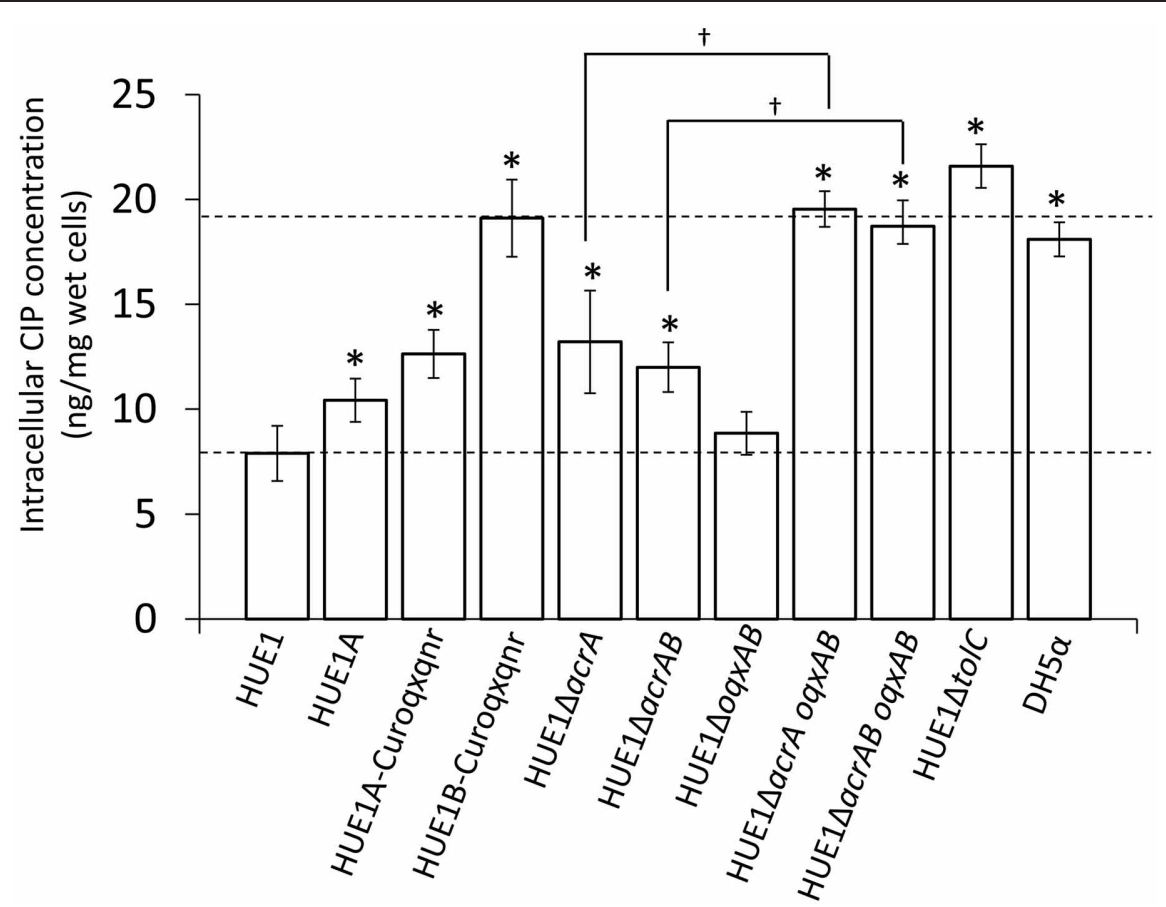

FIGURE 4 | Intracellular concentrations of CIP in HUE1 and its mutants obtained from plasmid curing and Red/ET recombination. Data represent the mean \pm standard deviation values of the results from three independent experiments. ${ }^{*} p<0.05$ compared to HUE1. ${ }^{\dagger} p<0.05$ between HUE1- $\triangle$ acrA

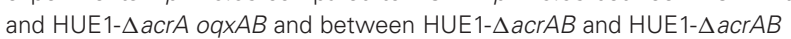
oqxAB, respectively.
AcrAB-TolC expression is controlled by several activators (i.e., soxS, marA, and rob) and repressors (i.e., acrR, $\operatorname{marR}$, and $\operatorname{sox} R$ ) (White et al., 1997; Poole, 2005; Keeney et al., 2008). HUE1 exhibited higher expression of $a c r A, a c r B$, tolC and these activators. In addition, HUE1 had concomitant disruption of the $a c r R$ and marR repressors. AcrR is a local repressor of AcrAB (Wang et al., 2001), and MarR is a repressor of MarA, which is one of the global activators of AcrAB (Keeney et al., 2008). Introduction of wild-type acrR or wild-type marR to HUE1 actually reduced acr $A$ and $a c r B$ mRNA expression and reduced fluoroquinolone MICs. These data suggested that overexpression of AcrAB-TolC in HUE1 was mediated by the concomitant disruptions of AcrR and MarR. However, other mechanisms are also responsible for the overexpression of AcrAB-TolC and other chromosomally 
encoded efflux pumps, since the group A series of HUE1 mutants was found to have only approximately half of the mRNA expression levels of chromosomal efflux pump genes, including those encoding $\mathrm{Acr} A \mathrm{~B}$, despite having the same disruptions in acrR and marR as HUE1. This will require further investigation.

Other chromosomally encoded efflux pumps, such as AcrEFTolC and AcrD, belonging to the RND family, MdtK (also known as YdhE or NorE), belonging to the multidrug and toxic compound extrusion family, and MdfA, belonging to the major facilitator superfamily, also excrete fluoroquinolones (Nishino and Yamaguchi, 2001; Poole, 2005; Nishino et al., 2006). The mRNA expression of the related genes were also higher in HUE1, and HUE1 knockout mutants of these genes exhibited reduced fluoroquinolone MICs compared to HUE1, although the influence of these knockouts was weaker than that of AcrAB. These results indicated that the fluoroquinolone-resistance mechanism in HUE1 was most likely associated with the overexpression of AcrAB-TolC, with minor contributions of other chromosomally encoded efflux pumps, in addition to $2 \mathrm{PMQRs}$, viz., oq $x A B$ and qnrS1.

OqxAB belongs to the RND-type efflux pump family, as does AcrAB (Hansen et al., 2004). Although OqxAB did not cause marked changes in fluoroquinolone MICs and intracellular CIP concentrations, we observed more distinct changes following acr $A B$ deletion. OqxAB requires TolC to excrete AMP, $\mathrm{CHL}$, and OLA, as does AcrAB (Hansen et al., 2004). In this study, knockout of tolC markedly decreased fluoroquinolone MICs and intracellular CIP concentrations and negated the effects of both $a c r A B$ and $o q \times A B$. This finding indicated that $O q x A B$, in conjunction with TolC, was also involved in mediating decreased fluoroquinolone susceptibility. TolC is therefore implicated in the functions of both

\section{REFERENCES}

Bin Kim, H., Wang, M. H., Park, C. H., Kim, E. C., Jacoby, G. A., and Hooper, D. C. (2009). oqxAB encoding a multidrug efflux pump in human clinical isolates of enterobacteriaceae. Antimicrobial Agents Chemother. 53, 3582-3584.

Bortolaia, V., Larsen, J., Damborg, P., and Guardabassi, L. (2011). Potential pathogenicity and host range of extended-spectrum betalactamase-producing Escherichia coli isolates from healthy poultry. Appl. Environ. Microbiol. 77, 5830-5833.

Breines, D. M., Ouabdesselam, S., Ng, E. Y., Tankovic, J., Shah, S., Soussy, C. J., et al. (1997). Quinolone resistance locus $\mathrm{nfxD}$ of Escherichia coli is a mutant allele of the parE gene encoding a subunit of topoisomerase IV. Antimicrob. Agents Chemother. 41, 175-179.

Cattoir, V., Poirel, L., Rotimi, V., Soussy, C. J., and Nordmann, P. (2007). Multiplex PCR for detection of plasmid-mediated quinolone resistance qnr genes in
ESBL-producing enterobacterial isolates. J. Antimicrob. Chemother. 60, 394-397.

Cesaro, A., Bettoni, R. R., Lascols, C., Mérens, A., Soussy, C. J., and Cambau, E. (2008). Low selection of topoisomerase mutants from strains of Escherichia coli harbouring plasmid-borne qnr genes. J. Antimicrob. Chemother. 61, 1007-1015. (2011). A fluoroquinoloneresistant Acinetobacter baumannii without the quinolone resistancedetermining region mutations. J. Antimicrob. Chemother. 66, 2668-2670.

Cizman, M., Orazem, A., KrizanHergouth, V., and Kolman, J. (2001). Correlation between increased consumption of fluoroquinolones in outpatients and resistance of Escherichia coli from urinary tract infections.

Clinical and Laboratory Standards Institute. (2011). Performance Standards for Antimicrobial
Chopra, S., and Galande, A. J. Antimicrob. Chemother. 47, 502.

$A c r A B$ and OqxAB. Although OqxAB minimally contributed to fluoroquinolone resistance in HUE1, it may largely contribute to supplementation of AcrAB functions. TolC is also required for the function of several efflux pumps (other than AcrAB), such as AcrEF and AcrD, in S. enterica serovar Typhimurium (Horiyama et al., 2010). Therefore, overexpression of TolC should also be an important element in the fluoroquinolone-resistance mechanism in HUE1 cells, as verified by our finding of the greatest reduction in fluoroquinolone MICs by knockout of tolC.

In conclusion, this study is the first to reveal the existence of a fluoroquinolone-resistance mechanism that is mediated without QRDR mutations in the E. coli clinical isolate, HUE1. This mechanism involved the concomitant presence of $o q \times A B$ and qnrS1 and was associated with the overexpression of AcrAB, other chromosomally encoded efflux pumps, and TolC. HUE1 was identified as phylogenic group A-O125:H37-ST48. This clonal group has also been isolated from humans and animals worldwide (Jorgensen et al., 2010; Bortolaia et al., 2011; Croxall et al., 2011). However, the susceptibilities of ST48 strains to fluoroquinolone compounds vary and have not yet been defined in all cases. Therefore, further epidemiological and molecular biology analyses of the ST48 lineage are required.

\section{ACKNOWLEDGMENTS}

The authors thank Hirotsugu Akizawa (Hokkaido University Hospital) for providing E. coli clinical isolates. This work was supported in part by a Grant-in-Aid from the Japanese Ministry of Health, Labour, and Welfare (H24-Shokuhin-Ippan-008) and a grant from the Program for Developing the Supporting System for Upgrading Education and Research from the Japan Ministry of Education, Culture, Sports, Science, and Technology.

Susceptibility Testing. CLSI M100S21. Wayne, PA: Clinical and Laboratory Standards Institute.

Conrad, S., Oethinger, M., Kaifel, K., Klotz, G., Marre, R., and Kern, W. V. (1996). GyrA mutations in highlevel fluoroquinolone-resistant clinical isolates of Escherichia coli. J. Antimicrob. Chemother. 38, 443-455.

Croxall, G., Hale, J., Weston, V., Manning, G., Cheetham, P., Achtman, M., et al. (2011). Molecular epidemiology of extraintestinal pathogenic Escherichia coli isolates from a regional cohort of elderly patients highlights the prevalence of ST131 strains with increased antimicrobial resistance in both community and hospital care settings. J. Antimicrob. Chemother. 66, 2501-2508.

Deane, S. M., and Rawlings, D. E. (2004). Plasmid evolution and interaction between the plasmid addiction stability systems of two related broad-host-range IncQlike plasmids. J. Bacteriol. 186, 2123-2133.
Edgar, R., Friedman, N., MolshanskiMor, S., and Qimron, U. (2012). Reversing bacterial resistance to antibiotics by phage-mediated delivery of dominant sensitive genes. Appl. Environ. Microbiol. 78, 744-751.

Elkins, C. A., and Nikaido, H. (2003). Chimeric analysis of AcrA function reveals the importance of its C-terminal domain in its interaction with the AcrB multidrug efflux pump. J. Bacteriol. 185, 5349-5356.

Ge, Q., Yamada, Y., and Zgurskaya, H. (2009). The C-terminal domain of AcrA is essential for the assembly and function of the multidrug efflux pump AcrAB-TolC. J. Bacteriol. 191, 4365-4371.

Hansen, L. H., Jensen, L. B., Sorensen, H. I., and Sorensen, S. J. (2007). Substrate specificity of the OqxAB multidrug resistance pump in Escherichia coli and selected enteric bacteria. J. Antimicrob. Chemother. 60, 145-147.

Hansen, L. H., Johannesen, E., Burmolle, M., Sorensen, A. H., and Sorensen, S. J. (2004). 
Plasmid-encoded multidrug efflux pump conferring resistance to olaquindox in Escherichia coli. Antimicrob. Agents Chemother. 48, 3332-3337.

Hata, M., Suzuki, M., Matsumoto, M., Takahashi, M., Sato, K., Ibe, S., et al. (2005). Cloning of a novel gene for quinolone resistance from a transferable plasmid in Shigella flexneri 2b. Antimicrob. Agents Chemother. 49, 801-803.

Heisig, P. (1996). Genetic evidence for a role of parC mutations in development of high-level fluoroquinolone resistance in Escherichia coli. Antimicrob. Agents Chemother. 40, 879-885.

Horiyama, T., Yamaguchi, A., and Nishino, K. (2010). TolC dependency of multidrug efflux systems in Salmonella enterica serovar Typhimurium. J. Antimicrob. Chemother. 65, 1372-1376.

Jacoby, G. A. (2005). Mechanisms of resistance to quinolones. Clin. Infect. Dis. 41, S120-S126.

Jorgensen, R. L., Nielsen, J. B., FriisMoller, A., Fjeldsoe-Nielsen, H., and Schonning, K. (2010). Prevalence and molecular characterization of clinical isolates of Escherichia coli expressing an AmpC phenotype. J. Antimicrob. Chemother. 65, 460-464.

Kado, C. I., and Liu, S. T. (1981). Rapid procedure for detection and isolation of Large and small Plasmids. J. Bacteriol. 145, 1365-1373.

Keeney, D., Ruzin, A., McAleese, F., Murphy, E., and Bradford, P. A. (2008). MarA-mediated overexpression of the AcrAB efflux pump results in decreased susceptibility to tigecycline in Escherichia coli. J. Antimicrob. Chemother. 61, 46-53.

Kobayashi, K., Tsukagoshi, N., and Aono, R. (2001). Suppression of hypersensitivity of Escherichia coli acrB mutant to organic solvents by integrational activation of the acrEF operon with the IS1 or IS2 element. J. Bacteriol. 183, 2646-2653.

Lindgren, P. K., Karlsson, A., and Hughes, D. (2003). Mutation rate and evolution of fluoroquinolone resistance in Escherichia coli isolates from patients with urinary tract infections. Antimicrob. Agents Chemother. 47, 3222-3232.

Nakaminami, H., Noguchi, N., and Sasatsu, M. (2010). Fluoroquinolone efflux by the plasmid-mediated multidrug efflux pump QacB variant QacBIII in Staphylococcus aureus. Antimicrob. Agents Chemother. 54, 4107-4111.

Nishino, K., Latifi, T., and Groisman, E. A. (2006). Virulence and drug resistance roles of multidrug efflux systems of Salmonella enterica serovar Typhimurium. Mol. Microbiol. 59, 126-141.

Nishino, K., and Yamaguchi, A. (2001). Analysis of a complete library of putative drug transporter genes in Escherichia coli. J. Bacteriol. 183, 5803-5812.

Okusu, H., Ma, D., and Nikaido, H. (1996). AcrAB efflux pump plays a major role in the antibiotic resistance phenotype of Escherichia coli multiple-antibiotic-resistance (MAR) mutants. J. Bacteriol. 178, 306-308.

Peña, C., Albareda, J. M., Pallares, R., Pujol, M., Tubau, F., and Ariza, J. (1995). Relationship between quinolone use and appearance of ciprofloxacin-resistant Escherichia coli in bloodstream infections. Antimicrob. Agents Chemother. 39, 520-524.

Poole, K. (2005). Efflux-mediated antimicrobial resistance. J. Antimicrob. Chemother. 56, 20-51.

Sanchez, G. V., Master, R. N., Karlowsky, J. A., and Bordon, J. M. (2012). In vitro antimicrobial resistance of urinary Escherichia coli isolates among U.S. outpatients from 2000 to 2010. Antimicrob. Agents Chemother. 56, 2181-2183.

Sato, T., Yokota, S., Uchida, I., Okubo, T., Ishihara, K., Fujii, N., et al. (2011). A fluoroquinolone-resistant Escherichia coli clinical isolate without quinolone resistancedetermining region mutations found in Japan. Antimicrob. Agents Chemother. 55, 3964-3965.

Sorensen, A. H., Hansen, L. H., Johannesen, E., and Sorensen, S. J. (2003). Conjugative plasmid conferring resistance to olaquindox. Antimicrob. Agents Chemother. 47, 798-799.

Tamamura, Y., Uchida, I., Tanaka, K., Okazaki, H., Tezuka, S., Hanyu, H., et al. (2011). Molecular epidemiology of salmonella enterica serovar typhimurium isolates from cattle in Hokkaido, Japan: evidence of clonal replacement and characterization of the disseminated clone. Appl. Environ. Microbiol. 77, 1739-1750.

Usui, M., Uchiyama, M., Iwanaka, M., Nagai, H., Yamamoto, Y., and Asai, T. (2009). Intracellular concentrations of enrofloxacin in quinolone-resistant Salmonella enterica subspecies enterica serovar Choleraesuis. Int. J. Antimicrob. Agents 34, 592-595.

Wang, H., Dzink-Fox, J. L., Chen, M., and Levy, S. B. (2001). Genetic characterization of highly fluoroquinolone-resistant clinical Escherichia coli strains from China: role of acrR mutations. Antimicrob. Agents Chemother. 45, 1515.39-1521.39.

White, D. G., Goldman, J. D., Demple, B., and Levy, S. B. (1997). Role of the acrAB locus in organic solvent tolerance mediated by expression of marA, soxS, or robA in Escherichia coli. J. Bacteriol. 179, 6122-6126.
Yoshida, H., Bogaki, M., Nakamura, M., Yamanaka, L. M., and Nakamura, S. (1991). Quinolone resistance-determining region in the DNA gyrase gyrB gene of Escherichia coli. Antimicrob. Agents Chemother. 35, 1647-1650.

Zhao, J., Chen, Z., Chen, S., Deng, Y., Liu, Y., Tian, W., et al. (2010). Prevalence and dissemination of oqxAB in Escherichia coli isolates from animals, farmworkers, and the environment. Antimicrob. Agents Chemother. 54, 4219-4224.

Conflict of Interest Statement: The authors declare that the research was conducted in the absence of any commercial or financial relationships that could be construed as a potential conflict of interest.

Received: 23 March 2013; accepted: 02 May 2013; published online: 24 May 2013.

Citation: Sato T, Yokota S-i, Uchida I, Okubo T, Usui M, Kusumoto M, Akiba $M$, Fujii $N$ and Tamura $Y$ (2013) Fluoroquinolone resistance mechanisms in an Escherichia coli isolate, HUE1, without quinolone resistancedetermining region mutations. Front. Microbiol. 4:125. doi: 10.3389/fmicb. 2013.00125

This article was submitted to Frontiers in Antimicrobials, Resistance and Chemotherapy, a specialty of Frontiers in Microbiology.

Copyright (c) 2013 Sato, Yokota, Uchida, Okubo, Usui, Kusumoto, Akiba, Fujii and Tamura. This is an open-access article distributed under the terms of the Creative Commons Attribution License, which permits use, distribution and reproduction in other forums, provided the original authors and source are credited and subject to any copyright notices concerning any third-party graphics etc. 